\title{
IDEMPOTENTS IN THE ENDOMORPHISM ALGEBRA OF A FINITE LATTICE
}

\author{
SERGE BOUC AND JACQUES THÉVENAZ
}

\begin{abstract}
We give a direct construction of a specific central idempotent in the endomorphism algebra of a finite lattice $T$. This idempotent is associated with all possible sublattices of $T$ which are totally ordered. A generalization is considered in a conjectural fashion and proved experimentally to hold in many cases.
\end{abstract}

Serge Bouc, CNRS-LAMFA, Université de Picardie - Jules Verne, 33, rue St Leu, F-80039 Amiens Cedex 1, France.

serge.bouc@u-picardie.fr

Corresponding author : Jacques Thévenaz, Institut de mathématiques, EPFL, Station 8, CH-1015 Lausanne, Switzerland.

jacques. thevenaz@epfl.ch

\section{Introduction}

Let $T$ be a finite lattice and let $\mathcal{E}$ be the monoid of all join-morphisms from $T$ to $T$. If $k$ is a commutative ring, the $k$-algebra of this monoid is the set $k \mathcal{E}$ of all $k$-linear combinations of elements of $\mathcal{E}$, with multiplication induced by bilinearity from the composition in $\mathcal{E}$. This $k$-algebra is of interest from a combinatorial point of view because it reflects the structure of $T$ in an algebraic fashion. Moreover, $k \mathcal{E}$ plays an important role in our work on correspondence functors [BT1, BT2, BT3, BT4, BT5], providing another motivation for the present work.

Let $k \mathcal{E}_{\text {tot }}$ be the two-sided ideal of $k \mathcal{E}$ spanned by the set $\mathcal{E}_{\text {tot }}$ of all joinendomorphisms whose image is totally ordered. We proved in [BT2] that $k \mathcal{E}_{\text {tot }}$ is generated by a central idempotent $e_{\text {tot }}$. In other words, there is a ring isomorphism

$$
k \mathcal{E} \cong k \mathcal{E}_{\text {tot }} \times \mathcal{D},
$$

where $\mathcal{D}$ is a complementary two-sided ideal. We also proved that $k \mathcal{E}_{\text {tot }}$ is isomorphic to a product of matrix algebras over $k$, hence semi-simple when $k$ is a field.

Unfortunately, the definition of $e_{\text {tot }}$ relies on some rather cumbersome constructions. In the present paper, we give a new point of view for this idempotent. We define an element $f_{\text {tot }} \in k \mathcal{E}$ by means of an easy and explicit formula (see Definition 2.2), which has the advantage of allowing for computer calculations and which does not depend on our previous work. Our main result is the following, which is proved in Section 5.

Date: November 3, 2021.

2010 Mathematics Subject Classification. 06A05, 06A07, 06A11, 06A12, 06B05, 16S99.

Key words and phrases. Poset, lattice, total order, idempotent. 


\subsection{Theorem.}

(a) $f_{\text {tot }}$ is a central idempotent of $k \mathcal{E}$.

(b) $f_{\text {tot }}$ is the identity element of the ideal $k \mathcal{E}_{\text {tot }}$.

This implies that $f_{\text {tot }}$ coincides with the idempotent $e_{\text {tot }}$ of our previous paper, but with a much easier definition. However, no improvement is made here concerning the semi-simplicity result mentioned above, which depends heavily on the methods of [BT2].

Pole lattices are generalizations of totally ordered lattices and are defined in Section 2. They play a role in our work on correspondence functors because they occur in the semi-simple part of the representation theory developed in [BT5]. Let $k \mathcal{E}_{\text {pole }}$ be the two-sided ideal of $k \mathcal{E}$ spanned by the set $\mathcal{E}_{\text {pole }}$ of all join-endomorphisms whose image is a pole lattice. We proved in [BT5] that $k \mathcal{E}_{\text {pole }}$ is generated by a central idempotent $e_{\text {pole }}$, so that there is a ring isomorphism

$$
k \mathcal{E} \cong k \mathcal{E}_{\text {pole }} \times \mathcal{D}^{\prime}
$$

for some two-sided ideal $\mathcal{D}^{\prime}$. Moreover, we proved that $k \mathcal{E}_{\text {pole }}$ is isomorphic to a product of matrix algebras over either $k$ or the group algebra of a group of order 2 , hence semi-simple when $k$ is a field of characteristic different from 2.

In the present paper, we give also a new point of view for the idempotent $e_{\text {pole }}$, but in a conjectural fashion. We define an element $f_{\text {pole }} \in k \mathcal{E}$ by means of a formula which is analogous to the definition of $f_{\text {tot }}$. We then conjecture the following :

\subsection{Conjecture.}

(a) $f_{\text {pole }}$ is a central idempotent of $k \mathcal{E}$.

(b) $f_{\text {pole }}$ is the identity element of the ideal $k \mathcal{E}_{\text {pole }}$.

In other words, we conjecture that $f_{\text {pole }}$ coincides with the idempotent $e_{\text {pole }}$ of our previous paper. Since the formula for $f_{\text {pole }}$ allows for computer calculations, we were able to verify that the conjecture holds in a very large number of cases.

The paper is organized as follows. Section 2 is introductory and contains the definition of $f_{\text {tot }}$ and $f_{\text {pole }}$. The formula for $f_{\text {tot }}$ is simplified in Sections 3 and 4 , while the main theorem is proved in Section 5. Section 6 contains an alternative proof of the equality $f_{\text {tot }}=e_{\text {tot }}$, using the original approach of [BT2]. Finally Section 7 deals with the conjecture in the case of pole lattices.

\section{Endomorphisms of finite lattices}

We first recall some basic facts we need about finite lattices. For the rest of this paper, $T$ denotes a finite lattice. Most constructions depend on $T$ but we usually do not include $T$ in the subsequent notation in order to keep it simple. We write $\leq$ for the partial order of $T, \vee$ for its join, $\wedge$ for its meet, $\hat{0}=\hat{0}_{T}$ for its least element, and $\hat{1}=\hat{1}_{T}$ for its greatest element. Recall that an empty join is equal to $\hat{0}$, while an empty meet is equal to $\hat{1}$. If $x \leq y$, we define the intervals

$$
[x, y]=\{z \in T \mid x \leq z \leq y\} \quad \text { and } \quad] x, y[=\{z \in T \mid x<z<y\} .
$$

Recall that, because $T$ is finite, the meet is uniquely determined by the join thanks to the finite expression

$$
x \wedge y=\bigvee_{\substack{a \in T \\ a \leq x, a \leq y}} a .
$$

In particular, any join-closed subposet $S$ of $T$ is a lattice in its own right, with a meet induced by its join, but this meet may not coincide with the meet of $T$. Note 
that $S$ contains $\hat{0}$ (the empty join), but not necessarily $\hat{1}$. We emphasize that we shall actually only use the join-semilattice structure of $T$.

If $T^{\prime}$ is another finite lattice, a join-morphism $\varphi: T \rightarrow T^{\prime}$ is a map such that, for any subset $X \subseteq T$, we have

$$
\varphi\left(\bigvee_{x \in X} x\right)=\bigvee_{x \in X} \varphi(x) .
$$

The case $X=\emptyset$ yields the property $\varphi(\hat{0})=\hat{0}$. However, a join-morphism need not respect the meet, and in particular need not map $\hat{1}$ to $\hat{1}$. We note that, whenever $\varphi: T \rightarrow T^{\prime}$ is a join-morphism, its image $\varphi(T)$ is a join-closed subposet of $T^{\prime}$, hence a lattice. We let $\mathcal{E}$ be the set of all join-endomorphisms of $T$. This is a monoid for the composition of maps and we let $k \mathcal{E}$ be the $k$-algebra of this monoid, where $k$ is a fixed commutative base ring.

Let $\mathcal{F}$ be a family of finite lattices, closed under the following two operations :

(a) If $S \in \mathcal{F}$ and $S^{\prime}$ is a join-closed subposet of $S$, then $S^{\prime} \in \mathcal{F}$.

(b) If $S \in \mathcal{F}$ and $\varphi: S \rightarrow S^{\prime}$ is a join-morphism, then $\varphi(S) \in \mathcal{F}$.

In particular, $\mathcal{F}$ is closed under taking images of join-endomorphisms :

$$
S \in \mathcal{F}, \alpha \in \mathcal{E} \Longrightarrow \alpha(S) \in \mathcal{F} \text {. }
$$

We define $\mathcal{E}_{\mathcal{F}}$ to be the subset of $\mathcal{E}$ consisting of all join-morphisms $\alpha: T \rightarrow T$ such that the image $\alpha(T)$ belongs to $\mathcal{F}$. We let $k \mathcal{E}_{\mathcal{F}}$ be the $k$-linear span of $\mathcal{E}_{\mathcal{F}}$ in $k \mathcal{E}$.

2.1. Lemma. $k \mathcal{E}_{\mathcal{F}}$ is a two-sided ideal of $k \mathcal{E}$.

Proof : Let $\alpha \in \mathcal{E}_{\mathcal{F}}$ and $\varphi \in \mathcal{E}$. Since $\varphi(T)$ is a join-closed subposet of $T$, it is clear that $\alpha \varphi(T)$ is a join-closed subposet of $\alpha(T)$, hence belongs to $\mathcal{F}$. Thus $\alpha \varphi \in \mathcal{E}_{\mathcal{F}}$. On the other hand, the lattice $\alpha(T)$ belongs to $\mathcal{F}$ and is mapped by $\varphi$ to $\varphi \alpha(T) \in \mathcal{F}$. Therefore $\varphi \alpha \in \mathcal{E}_{\mathcal{F}}$. The result follows by considering $k$-linear combinations.

The monoid $\mathcal{E}$ is partially ordered by defining, for any $\alpha, \beta \in \mathcal{E}$,

$$
\alpha \leq \beta \Longleftrightarrow \alpha(t) \leq \beta(t) \quad \forall t \in T .
$$

It is actually also a lattice via the join

$$
(\alpha \vee \beta)(t)=\alpha(t) \vee \beta(t),
$$

and the induced meet. We are interested in the subposet $\{\alpha \in \mathcal{E} \mid \alpha \geq$ id $\}$ and we note that, whenever $\alpha \geq$ id, the join-closed image $\alpha(T)$ contains $\hat{1}$ because $\alpha(\hat{1}) \geq \operatorname{id}(\hat{1})=\hat{1}$.

If $\overline{\mathcal{F}}$ is a family of finite lattices as above, we define

$$
\mathcal{H}_{\mathcal{F}}:=\{\alpha \in \mathcal{E} \mid \alpha \geq \mathrm{id}, \alpha(T) \in \mathcal{F}\}
$$

and consider its Möbius function $\mu_{\mathcal{H}}(\beta, \alpha)$, viewing $\mathcal{H}_{\mathcal{F}}$ as a full subposet of $\mathcal{E}$. We then define the element

$$
f_{\mathcal{F}}=\sum_{\alpha \in \mathcal{H}_{\mathcal{F}}} \sum_{\beta \in \mathcal{H}_{\mathcal{F}}} \mu_{\mathcal{H}_{\mathcal{F}}}(\beta, \alpha) \alpha \in k \mathcal{E}
$$

which will be our main concern throughout this paper. Since $\mu_{\mathcal{H}}(\beta, \alpha)=0$ whenever $\beta \not \leq \alpha$, the second sum actually runs over $\beta \in \mathcal{H}_{\mathcal{F}}$ such that $\beta \leq \alpha$. In that case, $\mu_{\mathcal{H}_{\mathcal{F}}}(\beta, \alpha)$ is equal to the reduced Euler characteristic $\tilde{\chi}(] \beta, \alpha\left[\mathcal{H}_{\mathcal{F}}\right)$ of the poset $] \beta, \alpha\left[\mathcal{H}_{\mathcal{F}}\right.$ of all endomorphisms $\gamma \in \mathcal{H}_{\mathcal{F}}$ such that $\beta<\gamma<\alpha$. It is convenient to add to $\mathcal{H}_{\mathcal{F}}$ an element $-\infty$, smaller than all elements of $\mathcal{H}_{\mathcal{F}}$, so that

$$
\sum_{\beta \in \mathcal{H}_{\mathcal{F}}} \mu_{\mathcal{H}_{\mathcal{F}}}(\beta, \alpha)=-\mu_{\mathcal{H}_{\mathcal{F}}}(-\infty, \alpha)
$$


The definition of $f_{\mathcal{F}}$ then takes the following form :

\subsection{Definition.}

$$
f_{\mathcal{F}}=-\sum_{\alpha \in \mathcal{H}_{\mathcal{F}}} \mu_{\mathcal{H}_{\mathcal{F}}}(-\infty, \alpha) \alpha
$$

We shall be first interested in $\mathcal{F}=$ tot, the family of totally ordered lattices, and the corresponding element $f_{\text {tot }} \in k \mathcal{E}$. Our main goal is to prove that $f_{\text {tot }}$ is a central idempotent of $k \mathcal{E}$ and that it generates the two-sided ideal $k \mathcal{E}_{\text {tot. }}$ To this end, we use a series of reductions of the above formula to a much simpler form. These reductions only work for the family of totally ordered lattices. They appear in Sections 3 and 4.

Later in Section 7, we shall also consider the family of pole lattices and the corresponding element $f_{\text {pole }} \in k \mathcal{E}$. Recall from [BT5] that a pole lattice is a finite lattice $P$ such that every $x \in P$ is comparable to every element of $P$ except at most one. In the latter case, if $x$ and $y$ are not comparable, then $y$ is called the twin of $x$ (and $x$ is in turn the twin of $y$ ). In other words, $x$ and $y$ have the same height and we have

$$
P=P_{<\{x, y\}} \sqcup\{x, y\} \sqcup P_{>\{x, y\}}
$$

Moreover,

$$
P_{<\{x, y\}}=P_{<x}=P_{<y}=[\hat{0}, x \wedge y] \quad \text { and } \quad P_{>\{x, y\}}=P_{>x}=P_{>y}=[x \vee y, \hat{1}] .
$$

Clearly, any totally ordered lattice is a pole lattice (with no twins). Some motivation for working with the family of pole lattices comes from our work on correspondence functors [BT5], because the part of this representation theory which is semi-simple is closely related to pole lattices.

To end this preliminary section, recall that the opposite partial order on the finite lattice $T$ yields the opposite lattice $T^{o p}$, swapping the role of $\vee$ and $\wedge$, and with $\hat{0}_{T^{o p}}=\hat{1}_{T}$ and $\hat{1}_{T^{o p}}=\hat{0}_{T}$. Associated with a join-morphism $\varphi: T \rightarrow T^{\prime}$, there is its opposite

$$
\varphi^{o p}: T^{o p} \longrightarrow T^{o p}, \quad \varphi^{o p}\left(t^{\prime}\right)=\bigvee_{\substack{t \in T \\ \varphi(t) \leq t^{\prime}}} t
$$

2.3. Lemma. Let $\varphi: T \rightarrow T^{\prime}$ be a join-morphism between two finite lattices.

(a) $\varphi^{o p}: T^{\prime o p} \rightarrow T^{o p}$ is a join-morphism (that is, a meet-morphism $T^{\prime} \rightarrow T$ ).

(b) $\left(\varphi^{o p}\right)^{o p}=\varphi$.

(c) If $t^{\prime} \in \varphi(T)$, then

$$
\varphi^{o p}\left(t^{\prime}\right)=\bigvee_{\substack{t \in T \\ \varphi(t)=t^{\prime}}} t=\sup \left\{t \in T \mid \varphi(t)=t^{\prime}\right\},
$$

and in particular $\varphi\left(\varphi^{o p}\left(t^{\prime}\right)\right)=t^{\prime}$.

(d) $\forall t \in T$ and $\forall t^{\prime} \in T^{\prime}$, we have

$$
\varphi(t) \leq t^{\prime} \Longleftrightarrow t \leq \varphi^{o p}\left(t^{\prime}\right) .
$$

Proof : See Lemma 8.1 and Property 8.3 in [BT2]. 


\section{Endomorphisms with a totally ordered image}

We consider the family $\mathcal{F}=$ tot of all totally ordered lattices. Recall that a chain in $T$ is a totally ordered subset of $T$. Recall also that

$$
\mathcal{H}_{\text {tot }}:=\{\alpha \in \mathcal{E} \mid \alpha \geq \mathrm{id}, \alpha(T) \in \text { tot }\} .
$$

Let $\sigma \in \mathcal{H}_{\text {tot }}$ and let $A:=\sigma(T)$ be its totally ordered image. Then $A$ is a chain containing $\hat{0}$ (because $\sigma(\hat{0})=\hat{0}$ ). Moreover, since $\sigma \geq \mathrm{id}$, the chain $A$ also contains $\hat{1}$, hence

$$
A=\left\{\hat{0}=a_{0}<a_{1}<\ldots<a_{n}=\hat{1}\right\}
$$

for some $n \geq 0$. We let $\mathcal{Z}_{n}$ be the set of all chains of size $(n+1)$ in $T$ whose least element is $\hat{0}$ and greatest element is $\hat{1}$ and we set $\mathcal{Z}:=\bigcup_{n \geq 0} \mathcal{Z}_{n}$. Letting

$$
b_{i}=\sigma^{o p}\left(a_{i}\right)=\sup \left\{t \in T \mid \sigma(t)=a_{i}\right\},
$$

we obtain a chain $B=\left\{\hat{0}=b_{0}<b_{1}<\ldots<b_{n}=\hat{1}\right\}$ ending with $\hat{1}$ (because $\sigma^{o p}(\hat{1})=\hat{1}$ ) and starting with $\hat{0}$ (because id $\leq \sigma$, hence $\left.b_{0} \leq \sigma\left(b_{0}\right)=\hat{0}\right)$. In other words, $A, B$ belong to $\mathcal{Z}_{n}$ and $\sigma$ is characterized by the rule

$$
\sigma(t)=a_{i} \Longleftrightarrow t \leq b_{i} \text { and } t \not \leq b_{i-1} \text {. }
$$

In particular, we have

$$
b_{i} \leq a_{i} \text { for all } 0 \leq i \leq n,
$$

because $b_{i}=\operatorname{id}\left(b_{i}\right) \leq \sigma\left(b_{i}\right)=a_{i}$.

We now show the converse.

3.3. Lemma. Given two chains of the same length $A, B \in \mathcal{Z}_{n}$ and satisfying (3.2), the rule (3.1) defines a join-endomorphism $\sigma \in \mathcal{H}_{\text {tot }}$.

Proof : The rule (3.1) defines a map $\sigma: T \rightarrow T$ whose image is $A$. Let $s, t \in T$. Since $A=\sigma(T)$ is totally ordered, we can assume that $\sigma(s) \leq \sigma(t)$. We let $a_{i}=\sigma(s)$ and $a_{j}=\sigma(t)$, so that $i \leq j$. In particular, $b_{i} \leq b_{j}$. The rule (3.1) implies that $s \leq b_{i}$ and that $t \leq b_{j}, t \not \leq b_{j-1}$. It follows that $s \vee t \leq b_{j}$ and $s \vee t \not \leq b_{j-1}$. Therefore $\sigma(s \vee t)=a_{j}=\sigma(t)=\sigma(s) \vee \sigma(t)$. Since we also have $\sigma(\hat{0})=\hat{0}$ by (3.1), this shows that $\sigma$ is a join-endomorphism. Finally (3.1) and (3.2) imply that $\sigma \geq$ id. It follows that $\sigma \in \mathcal{H}_{\text {tot }}$.

3.4. Corollary. The set $\mathcal{H}_{\text {tot }}$ is parametrized by the set of pairs $(A, B)$ of chains $A, B \in \mathcal{Z}$ of the same length and satisfying (3.2).

We write $\sigma_{B}^{A}$ for the endomorphism parametrized by $A$ and $B$. We first show that the case $A=B$ plays a special role.

3.5. Lemma. Let $A, B \in \mathcal{Z}_{n}$ satisfying (3.2) and let $\sigma=\sigma_{B}^{A}$. Then $\sigma^{2}=\sigma$ if and only if $A=B$.

Proof: Suppose that $\sigma^{2}=\sigma$. Since $\sigma\left(b_{i}\right)=a_{i}$, we get $\sigma\left(a_{i}\right)=\sigma^{2}\left(b_{i}\right)=\sigma\left(b_{i}\right)=a_{i}$, hence

$$
a_{i} \leq \sup \left\{t \in T \mid \sigma(t)=a_{i}\right\}=b_{i} \leq a_{i},
$$

using (3.2). Therefore $a_{i}=b_{i}$, hence $A=B$.

Suppose now that $A=B$. Since $\sigma\left(b_{i}\right)=a_{i}$, we get $\sigma\left(a_{i}\right)=a_{i}$. Now, for any $t \in T$, we have $\sigma(t)=a_{i}$ for some $i$, hence $\sigma^{2}(t)=\sigma\left(a_{i}\right)=a_{i}$. This shows that $\sigma^{2}=\sigma$. 
In the case $A=B$, we write simply $\sigma_{A}:=\sigma_{A}^{A}$. By the rule (3.1), the map $\sigma_{A}$ can be described directly by the following formula :

$$
\sigma_{A}(t)=\min \{a \in A \mid t \leq a\} .
$$

Because of its importance in what follows, we introduce another useful notation : $\mathcal{G}_{\text {tot }}:=\left\{\sigma \in \mathcal{H}_{\text {tot }} \mid \sigma^{2}=\sigma\right\}, \quad$ i.e. $\quad \mathcal{G}_{\text {tot }}:=\left\{\sigma \in \mathcal{E} \mid \sigma \geq \mathrm{id}, \sigma(T) \in\right.$ tot,$\left.\sigma^{2}=\sigma\right\}$. By Corollary 3.4 and Lemma 3.5, the set $\mathcal{G}_{\text {tot }}$ is parametrized by the set of chains $A \in \mathcal{Z}$, noticing that any pair $(A, A)$ obviously satisfies Condition (3.2). The endomorphism parametrized by $A$ is $\sigma_{A}$ and therefore

$$
\mathcal{G}_{\text {tot }}=\left\{\sigma_{A} \mid A \in \mathcal{Z}\right\} \text {. }
$$

In the definition of $f_{\text {tot }}$ given by (2.2), the sum runs over all $\alpha \in \mathcal{H}_{\text {tot }}$ and they have the form $\alpha=\sigma_{B}^{A}$ for some chains $A, B \in \mathcal{Z}$ satisfying (3.2). Our first main result asserts that many such terms have a zero coefficient in the expression of $f_{\text {tot }}$.

3.8. Theorem. Let $A, B \in \mathcal{Z}$ satisfying (3.2).

(a) If $A=B$, we have $\mu_{\mathcal{G}_{\text {tot }}}\left(-\infty, \sigma_{A}\right)=\mu_{\mathcal{H}_{\text {tot }}}\left(-\infty, \sigma_{A}\right)$.

(b) If $A \neq B$, we have $\mu_{\mathcal{H}_{\text {tot }}}\left(-\infty, \sigma_{B}^{A}\right)=0$.

This will be proved in Section 4. It follows from (b) that the sum in the expression of $f_{\text {tot }}$ (in Definition 2.2) only runs over endomorphisms $\sigma_{A}$ where $A \in \mathcal{Z}$. Therefore, using (a), we obtain

$$
f_{\text {tot }}=-\sum_{A \in \mathcal{Z}} \mu_{\mathcal{H}_{\text {tot }}}\left(-\infty, \sigma_{A}\right) \sigma_{A}=-\sum_{A \in \mathcal{Z}} \mu_{\mathcal{G}_{\text {tot }}}\left(-\infty, \sigma_{A}\right) \sigma_{A} .
$$

The set $\mathcal{Z}$ is partially ordered by inclusion. It has no greatest element (unless $T$ is totally ordered) and we let $\infty$ be an additional element, larger than any $A \in \mathcal{Z}$.

3.10. Lemma. Consider the map

$$
\theta: \mathcal{Z} \sqcup\{\infty\} \longrightarrow\{-\infty\} \sqcup \mathcal{G}_{\text {tot }}, \quad \theta(A)=\sigma_{A} \text { and } \theta(\infty)=-\infty .
$$

(a) $\theta$ is an anti-isomorphism of posets : $A \subseteq B \Longleftrightarrow \sigma_{A} \geq \sigma_{B}$.

(b) For any $A \in \mathcal{Z}$, we have $\mu_{\mathcal{G}_{\text {tot }}}\left(-\infty, \sigma_{A}\right)=\mu_{\mathcal{Z}}(A, \infty)$.

Proof : By Corollary 3.4 and Lemma 3.5, the map $\theta$ is a bijection. Let $A, B \in \mathcal{Z}$ and suppose first that $A \subseteq B$. Let $t \in T$ and set $a=\sigma_{A}(t)$, so in particular $t \leq a$. Then $a \in A$, hence $a \in B$, and so $\sigma_{B}(a)=a$. Thus

$$
\sigma_{B}(t) \leq \sigma_{B}(a)=a=\sigma_{A}(t)
$$

and therefore $\sigma_{B} \leq \sigma_{A}$.

Assume now that $\sigma_{B} \leq \sigma_{A}$ and let $a \in A$. Then

$$
a \leq \sigma_{B}(a) \leq \sigma_{A}(a)=a,
$$

hence $\sigma_{B}(a)=a$, which implies that $a \in B$. Therefore $A \subseteq B$.

Statement (b) is an immediate consequence of (a).

Together with (3.9), Lemma 3.10 yields the following corollary :

3.11. Corollary. $f_{\text {tot }}=-\sum_{A \in \mathcal{Z}} \mu_{\mathcal{Z}}(A, \infty) \sigma_{A}$.

From now on, we shall work with this much simpler expression of $f_{\text {tot }}$. For later use, we show that the Möbius function of $\mathcal{Z}$ can be expressed in terms of the Möbius function of $T$. 
3.12. Lemma. Let $A=\left\{\hat{0}=a_{0}<a_{1}<\ldots<a_{n}=\hat{1}\right\}$ be an element of $\mathcal{Z}_{n}$. Then

$$
\mu_{\mathcal{Z}}(A, \infty)=(-1)^{n+1} \prod_{k=1}^{n} \mu_{T}\left(a_{k-1}, a_{k}\right),
$$

where $\mu_{T}\left(a_{k-1}, a_{k}\right)$ denotes the Möbius function of $T$.

Proof : For any poset $X$, let $s_{i}(X)$ be the number of chains of cardinality $i$ in $X$. For $i=0$, there is the empty chain, so $s_{0}(X)=1$. It is well-known that

$$
\mu_{\mathcal{Z}}(A, \infty)=\tilde{\chi}(] A, \infty[)=\sum_{i \geq 0}(-1)^{i-1} s_{i}(] A, \infty[)
$$

The sign is $(-1)^{i-1}$ because a chain of cardinality $i$ is an $(i-1)$-simplex. Now if $A^{\prime}$ is a chain with $A \subseteq A^{\prime}$, then $A^{\prime}$ is obtained from $A$ by inserting a chain in each interval $] a_{k-1}, a_{k}$ [ independently. Therefore

$$
s_{i}(] A, \infty[)=\sum_{\substack{i_{1}, \ldots, i_{n} \geq 0 \\ i_{1}+\ldots+i_{n}=i}} \prod_{k=1}^{n} s_{i_{k}}(] a_{k-1}, a_{k}[)
$$

and it follows that

$$
\begin{aligned}
\mu_{\mathcal{Z}}(A, \infty) & =\sum_{i_{1}, \ldots, i_{n} \geq 0}(-1)^{i_{1}+\ldots+i_{n}-1} \prod_{k=1}^{n} s_{i_{k}}(] a_{k-1}, a_{k}[) \\
& =(-1) \prod_{k=1}^{n}\left(\sum_{i_{k} \geq 0}(-1)^{i_{k}} s_{i_{k}}(] a_{k-1}, a_{k}[)\right. \\
& =(-1)^{n+1} \prod_{k=1}^{n}\left(\sum_{i_{k} \geq 0}(-1)^{i_{k}-1} s_{i_{k}}(] a_{k-1}, a_{k}[)\right. \\
& =(-1)^{n+1} \prod_{k=1}^{n} \mu_{T}\left(a_{k-1}, a_{k}\right)
\end{aligned}
$$

as was to be shown.

3.13. Remark. In the expression of $f_{\text {tot }}$ in Corollary 3.11, the sum could be restricted to all $A \in \mathcal{Z}$ such that the lattice $\left[a_{k-1}, a_{k}\right]$ is complemented for each $k=$ $1, \ldots, n$, where $A=\left\{\hat{0}=a_{0}<a_{1}<\ldots<a_{n}=\hat{1}\right\}$. This is because

$$
\mu_{\mathcal{Z}}(a, \infty)=(-1)^{n+1} \prod_{k=1}^{n} \mu_{T}\left(a_{k-1}, a_{k}\right)
$$

by Lemma 3.12 and $\mu_{T}\left(a_{k-1}, a_{k}\right)=0$ whenever the lattice $\left[a_{k-1}, a_{k}\right]$ is not complemented, by Crapo's formula (see Exercice 92 of Chapter 3 in [St]).

\section{Proof of Theorem 3.8}

Throughout this section, we simplify notation and define $\mathcal{H}=\mathcal{H}_{\text {tot }}$ and $\mathcal{G}=\mathcal{G}_{\text {tot }}$. By Corollary 3.4, the elements of $\mathcal{H}$ have the form $\sigma_{B}^{A}$ where $A, B \in \mathcal{Z}$ satisfy (3.2). By (3.7), the elements of $\mathcal{G}$ have the form $\sigma_{A}:=\sigma_{A}^{A}$ where $A \in \mathcal{Z}$. For any $\tau \in \mathcal{H}$, we also write $\mathcal{G}_{\leq \tau}:=\{\sigma \in \mathcal{G} \mid \sigma \leq \tau\}$ and $\mathcal{G}_{<\tau}:=\{\sigma \in \mathcal{G} \mid \sigma<\tau\}$. We shall use some of Quillen's basic results on homotopy of posets from [Qu]. 
4.1. Theorem. For any $\lambda \in \mathcal{H}-\mathcal{G}$, the poset $\mathcal{G}_{\leq \lambda}$ is contractible.

Before proving Theorem 4.1, we first show that Theorem 3.8 follows easily from Theorem 4.1.

4.2. Lemma. Let $\tau \in \mathcal{H}$.

(a) The inclusion map $\mathcal{G}_{<\tau} \hookrightarrow \mathcal{H}_{<\tau}$ is a homotopy equivalence.

(b) If $\tau \in \mathcal{G}$, we have $\mu_{\mathcal{G}}(-\infty, \tau)=\mu_{\mathcal{H}}(-\infty, \tau)$.

(c) If $\tau \notin \mathcal{G}$, we have $\mu_{\mathcal{H}}(-\infty, \tau)=0$.

Proof : (a) For any $\sigma \in \mathcal{H}_{<\tau}$, the poset $\mathcal{G}_{\leq \sigma}$ is contractible. This is obvious if $\sigma \in \mathcal{G}$ because $\mathcal{G}_{\leq \sigma}$ is a cone on $\sigma$, while this follows from Theorem 4.1 if $\sigma \notin \mathcal{G}$. Thus Proposition 1.6 in [Qu] applies and the inclusion map $\mathcal{G}_{<\tau} \hookrightarrow \mathcal{H}_{<\tau}$ is a homotopy equivalence.

(b) This follows from (a) by taking reduced Euler characteristics.

(c) We have $\mathcal{G}_{<\tau}=\mathcal{G}_{\leq \tau}$ because $\tau \notin \mathcal{G}$. Thus $\mathcal{G}_{<\tau}$ is contractible by Theorem 4.1 and so is $\mathcal{H}_{<\tau}$ by the homotopy equivalence of part (a). Therefore the reduced Euler characteristic of $\mathcal{H}_{<\tau}$ is zero, in other words the Möbius function in (c) vanishes. $\square$

Proof of Theorem 3.8 : By Corollary 3.4, any $\tau \in \mathcal{H}$ has the form $\tau=\sigma_{B}^{A}$ for $A, B \in \mathcal{Z}$ satisfying (3.2). Moreover, $\tau \in \mathcal{G}$ if and only if $A=B$, by Lemma 3.5. Now parts (a) and (b) of Theorem 3.8 are exactly parts (b) and (c) of Lemma 4.2, written differently.

Proof of Theorem 4.1 : Using Corollary 3.4 and Lemma 3.5, we fix $\lambda=\sigma_{B}^{A}$ with $A \neq B$ and we write

$$
A=\left\{\hat{0}=a_{0}<a_{1}<\ldots<a_{n}=\hat{1}\right\} \text { and } B=\left\{\hat{0}=b_{0}<b_{1}<\ldots<b_{n}=\hat{1}\right\} .
$$

For any $1 \leq k \leq n$, replace $\left\{b_{k}, \ldots, b_{n}\right\}$ by $\left\{a_{k}, \ldots, a_{n}\right\}$ and define

$$
B_{k}=\left\{\hat{0}=b_{0}<\ldots<b_{k-1}<a_{k}<\ldots<a_{n}=\hat{1}\right\} .
$$

Define $\lambda_{k}=\sigma_{B_{k}}^{A}$ and notice that $\lambda_{n}=\sigma_{B}^{A}=\lambda$ and $\lambda_{1}=\sigma_{A}^{A}=\sigma_{A} \in \mathcal{G}$. Using (3.1), it is elementary to check that

$$
\lambda_{k} \leq \lambda_{k+1}, \forall 1 \leq k \leq n-1 .
$$

Our strategy is to pass from $\mathcal{G}_{\leq \lambda_{n}}$ to the single point $\left\{\lambda_{1}\right\}$ by a sequence of homotopy equivalences.

We first need to characterize the elements of $\mathcal{G}_{\leq \lambda_{k}}$.

4.4. Lemma. Let $C \in \mathcal{Z}$ and $1 \leq k \leq n$.

$$
\sigma_{C} \in \mathcal{G}_{\leq \lambda_{k}} \Longleftrightarrow\left\{\begin{array}{l}
\text { if } 1 \leq i \leq k-1 \text { and } t \leq b_{i}, \text { then } \sigma_{C}(t) \leq a_{i} . \\
a_{k}, \ldots, a_{n} \in C \text {. }
\end{array}\right.
$$

Proof : Suppose that $\sigma_{C} \leq \lambda_{k}$. If $t \leq b_{i}$ with $i \leq k-1$, then $\sigma_{C}(t) \leq \lambda_{k}(t) \leq$ $\lambda_{k}\left(b_{i}\right)=a_{i}$. If $i \geq k$, then since id $\leq \sigma_{C}$, we have $a_{i} \leq \sigma_{C}\left(a_{i}\right) \leq \lambda_{k}\left(a_{i}\right)=a_{i}$, hence $\sigma_{C}\left(a_{i}\right)=a_{i}$, that is, $a_{i} \in C$.

Suppose conversely that the right hand side conditions hold. If $1 \leq i \leq k-1$, $t \leq b_{i}$, and $t \not \leq b_{i-1}$, then $\sigma_{C}(t) \leq a_{i}=\lambda_{k}(t)$ by applying the rule (3.1) to $\lambda_{k}$. If $k \leq i \leq n, t \leq a_{i}, t \not \leq a_{i-1}$ (respectively $t \not \leq b_{k-1}$ in case $i=k$ ), then $a_{i} \in C$ and therefore $\sigma_{C}(t) \leq \sigma_{C}\left(a_{i}\right)=a_{i}=\lambda_{k}(t)$, by applying again the rule (3.1) to $\lambda_{k}$. 
For any chain $C$ satisfying $a_{k}, \ldots, a_{n} \in C$ and for any $k \leq i \leq n$, we let

$$
C_{i}:=\left\{c \in C \mid c<a_{i}, c \not \leq a_{i-1}\right\} \text { and } C_{\not \leq a_{k-1}}:=\left\{c \in C \mid c \not \leq a_{k-1}\right\} .
$$

It should be noticed that such a chain $C$ may be (much) longer than $A$, because $\left.C_{i}=C \cap\right] a_{i-1}, a_{i}[$ if $i \geq k+1$ and this may be nonempty. For any $1 \leq k \leq n$, define now

$$
\mathcal{G}_{\leq \lambda_{k}}^{\prime}=\left\{\sigma_{C} \in \mathcal{G}_{\leq \lambda_{k}} \mid C_{\not \leq a_{k-1}}=\left\{a_{k}, \ldots, a_{n}\right\}\right\},
$$

In other words, $\sigma_{C} \in \mathcal{G}_{\leq \lambda_{k}}^{\prime}$ if and only if $C_{i}=\emptyset$ for any $k \leq i \leq n$. It follows that, whenever $\sigma_{C} \in \mathcal{G}_{\leq \lambda_{k}}^{\prime}$, we obtain

$$
k \leq i \leq n, t \leq a_{i}, t \not \leq a_{i-1} \Longrightarrow \sigma_{C}(t)=a_{i},
$$

(whereas (3.6) only implies an inequality $\sigma_{C}(t) \leq a_{i}$ when $\left|C_{i}\right| \geq 2$ ). Clearly, (4.3) implies that $\mathcal{G}_{\leq \lambda_{k}} \subseteq \mathcal{G}_{\leq \lambda_{k+1}}$ and $\mathcal{G}_{\leq \lambda_{k}}^{\prime} \subseteq \mathcal{G}_{\leq \lambda_{k+1}}^{\prime}$ for any $k<n$. It follows that we have inclusions

Now define

$$
\mathcal{G}_{\leq \lambda_{k}}^{\prime} \subseteq \mathcal{G}_{\leq \lambda_{k}} \cap \mathcal{G}_{\leq \lambda_{k+1}}^{\prime} \subseteq \mathcal{G}_{\leq \lambda_{k}}
$$

and similarly, for $1 \leq k \leq n-1$,

$$
\rho_{n}: \mathcal{G}_{\leq \lambda_{n}} \longrightarrow \mathcal{G}_{\leq \lambda_{n}}^{\prime}, \quad \rho_{n}\left(\sigma_{C}\right)=\sigma_{C-C_{n}},
$$

$$
\rho_{k}: \mathcal{G}_{\leq \lambda_{k}} \cap \mathcal{G}_{\leq \lambda_{k+1}}^{\prime} \longrightarrow \mathcal{G}_{\leq \lambda_{k}}^{\prime}, \quad \rho_{k}\left(\sigma_{C}\right)=\sigma_{C-C_{k}} .
$$

4.5. Lemma. $\rho_{k}$ is a map of posets and is a homotopy equivalence.

Proof : If $\sigma_{C} \in \mathcal{G}_{\leq \lambda_{k}} \cap \mathcal{G}_{\leq \lambda_{k+1}}^{\prime}$ (respectively $\sigma_{C} \in \mathcal{G}_{\leq \lambda_{n}}$ if $k=n$ ), then $C_{i}=\emptyset$ for any $i>k$ and therefore $\left(\bar{C}-C_{k}\right)_{i}=\emptyset$ for any $i \geq k$. It follows that $\sigma_{C-C_{k}} \in \mathcal{G}_{\leq \lambda_{k}}^{\prime}$ and so the map $\rho_{k}$ is well-defined. It is order-preserving because, by Lemma 3.10 ,

$$
\sigma_{C} \geq \sigma_{C^{\prime}} \Rightarrow C \subseteq C^{\prime} \Rightarrow C-C_{k} \subseteq C^{\prime}-C_{k}^{\prime} \Rightarrow \sigma_{C-C_{k}} \geq \sigma_{C^{\prime}-C_{k}^{\prime}},
$$

using the fact that $C_{k}^{\prime} \cap C=C_{k}$.

Since $C-C_{k} \subseteq C$, we have $\rho_{k}\left(\sigma_{C}\right) \geq \sigma_{C}$. Therefore, if $j: \mathcal{G}_{\leq \lambda_{k}}^{\prime} \rightarrow \mathcal{G}_{\leq \lambda_{k}} \cap \mathcal{G}_{\leq \lambda_{k+1}}^{\prime}$ (respectively $j: \mathcal{G}_{\leq \lambda_{k}}^{\prime} \rightarrow \mathcal{G}_{\leq \lambda_{k}}$ if $k=n$ ) denotes the inclusion map, then $\rho_{k} j=$ id and $j \rho_{k} \geq \mathrm{id}$. By Property 1.3 in [Qu], $j \rho_{k}$ is homotopic to id and it follows that $\rho_{k}$ is a homotopy equivalence.

Now for $2 \leq k \leq n$, define

$$
\pi_{k}: \mathcal{G}_{\leq \lambda_{k}}^{\prime} \longrightarrow \mathcal{G}_{\leq \lambda_{k-1}} \cap \mathcal{G}_{\leq \lambda_{k}}^{\prime}, \quad \pi_{k}\left(\sigma_{C}\right)=\sigma_{C \cup\left\{a_{k-1}\right\}} .
$$

4.6. Lemma. $\pi_{k}$ is a map of posets and is a homotopy equivalence.

Proof : Let $\sigma_{C} \in \mathcal{G}_{\leq \lambda_{k}}^{\prime}$, so that $C_{\not a_{k-1}}=\left\{a_{k}, \ldots, a_{n}\right\}$. We first prove that $C \cup\left\{a_{k-1}\right\}$ is totally ordered (i.e. a chain in $\mathcal{Z}$ ), so that $\sigma_{C \cup\left\{a_{k-1}\right\}}$ makes sense. We must show that any $c \in C$ is comparable to $a_{k-1}$. This is clear if $c \leq a_{k-1}$, so we may assume that $c \not \leq a_{k-1}$. Then $c=a_{i}$ for some $i \geq k$ by the definition of $\mathcal{G}_{\leq \lambda_{k}}^{\prime}$, hence $c=a_{i}>a_{k-1}$.

Now we get $a_{k-1}, a_{k}, \ldots, a_{n} \in C \cup\left\{a_{k-1}\right\}$ and Lemma 4.4 implies that $\sigma_{C \cup\left\{a_{k-1}\right\}} \in$ $\mathcal{G}_{\leq \lambda_{k-1}}$. It follows that $\sigma_{C \cup\left\{a_{k-1}\right\}} \in \mathcal{G}_{\leq \lambda_{k-1}} \cap \mathcal{G}_{\leq \lambda_{k}}^{\prime}$ and the map $\pi_{k}$ is well-defined. It is order-preserving, by using Lemma 3.10.

Since $C \cup\left\{a_{k-1}\right\} \supseteq C$, we have $\pi_{k}\left(\sigma_{C}\right) \leq \sigma_{C}$. Therefore, if $j: \mathcal{G}_{\leq \lambda_{k-1}} \cap \mathcal{G}_{\leq \lambda_{k}}^{\prime} \rightarrow$ $\mathcal{G}_{\leq \lambda_{k}}^{\prime}$ denotes the inclusion map, then $\pi_{k} j=$ id and $j \pi_{k} \leq$ id. By Property 1.3 in $[\mathrm{Qu}], j \pi_{k}$ is homotopic to id and it follows that $\pi_{k}$ is a homotopy equivalence, as was to be shown. 
Now the sequence of homotopy equivalences $\rho_{n}, \pi_{n}, \rho_{n-1}, \pi_{n-1}, \ldots, \rho_{2}, \pi_{2}, \rho_{1}$ shows that $\mathcal{G}_{\leq \lambda_{n}}$ is homotopy equivalent to $\mathcal{G}_{\leq \lambda_{1}}^{\prime}$. But for any $\sigma_{C} \in \mathcal{G}_{\leq \lambda_{1}}^{\prime}$, we have

$$
C=\left\{\hat{0}=a_{0}<a_{1}<\ldots<a_{n}=\hat{1}\right\}=A,
$$

so $\sigma_{C}=\sigma_{A}=\lambda_{1}$. Thus $\mathcal{G}_{\leq \lambda_{1}}^{\prime}$ is a singleton, hence contractible, and therefore $\mathcal{G}_{\leq \lambda_{n}}$ is contractible as well. This completes the proof of Theorem 4.1.

\section{The main theorem}

In this section, we prove the main properties of the element $f_{\text {tot }}$, establishing the connection between $f_{\text {tot }}$ and the two-sided ideal $k \mathcal{E}_{\text {tot }}$ of the $k$-algebra $k \mathcal{E}$. In order to simplify notation throughout this section, we write $\mu$ instead of $\mu_{\mathcal{Z}}$ and $\sigma_{A}$ instead of $\sigma_{A}^{A}$.

5.1. Theorem. Let $f_{\text {tot }}$ be as in Corollary 3.11.

(a) $f_{\text {tot }}$ is a central idempotent of $k \mathcal{E}$.

(b) $f_{\text {tot }}$ is the identity element of the ideal $k \mathcal{E}_{\text {tot }}$.

The proof depends on a main lemma.

5.2. Lemma. $f_{\text {tot }} \psi=\psi$, for all $\psi \in \mathcal{E}_{\text {tot }}$.

Proof : By Corollary 3.11, recall that $f_{\text {tot }}=-\sum_{A \in \mathcal{Z}} \mu(A, \infty) \sigma_{A}$. Let $X=\operatorname{Im}(\psi)$, which is totally ordered because $\psi \in \mathcal{E}_{\text {tot }}$. We decompose $\psi$ as the composite of a surjective join-morphism $T \rightarrow X$ followed by the inclusion map $i_{X}: X \rightarrow T$. It suffices to prove that $f_{\text {tot }} i_{X}=i_{X}$ for any chain $X$ in $T$ starting with $\hat{0}$. Note that $f_{\text {tot }} i_{X}$ is a $k$-linear combination of maps from $X$ to $T$, so all the following computations must take place in the $k$-linear span of all maps $X \rightarrow T$. Now we have

$$
f_{\text {tot }} i_{X}=-\sum_{A \in \mathcal{Z}} \mu(A, \infty) \sigma_{A} i_{X}=\sum_{\varphi: X \rightarrow T}\left(-\sum_{\substack{A \in \mathcal{Z} \\ \sigma_{A} i_{X}=\varphi}} \mu(A, \infty)\right) \varphi .
$$

By the definition of $\sigma_{A}$, the equation $\sigma_{A} i_{X}=\varphi$ means that, for any $x \in X$, the element $\varphi(x)$ is the least element of $A$ such that $\varphi(x) \geq x$. In other words, the condition $\sigma_{A} i_{X}=\varphi$ is equivalent to

$$
[x, \varphi(x)] \cap A=\{\varphi(x)\}, \forall x \in X .
$$

Any map $\varphi: X \rightarrow T$ appearing in the sum (5.3) must satisfy the following 3 conditions :

(a) $\varphi$ is order-preserving and $\varphi(\hat{0})=\hat{0}$ (that is, $\varphi$ is a join-morphism).

(b) $\varphi(x) \geq x$, for all $x \in X$.

(c) If $x, y \in X$ satisfy $x \leq y \leq \varphi(x)$, then $\varphi(y)=\varphi(x)$.

In order to prove this, we note that the coefficient of $\varphi$ in (5.3) is nonzero only if there exists at least one $A \in \mathcal{Z}$ such that $\sigma_{A} i_{X}=\varphi$. Condition (a) follows from the fact that both $i_{X}$ and $\sigma_{A}$ are order-preserving and map $\hat{0}$ to $\hat{0}$, hence $\varphi=\sigma_{A} i_{X}$ has the same properties. Condition (b) is clear because $\sigma_{A} \geq \mathrm{id}$. For condition (c), note that the only element of $[x, \varphi(x)] \cap A$ is $\varphi(x)$, so the definition of $\sigma_{A}$ yields $\sigma_{A}(y)=\varphi(x)$, that is, $\varphi(y)=\varphi(x)$.

Now we prove that, if $\varphi$ satisfies (b) and (c), then (5.4) is equivalent to

$$
\left(\bigcup_{x \in X}[x, \varphi(x)]\right) \cap A=\varphi(X) \text {. }
$$


It is clear that (5.4) implies (5.5). Assume now (5.5) and let $x \in X$. Notice that $[x, \varphi(x)] \cap A$ is nonempty because $\varphi(x) \in \varphi(X)$, hence $\varphi(x) \in A$ by (5.5), and so $\varphi(x) \in[x, \varphi(x)] \cap A$. For any $b \in[x, \varphi(x)] \cap A$, we have $b \in \varphi(X)$ by $(5.5)$, that is, $b=\varphi(y)$ for some $y \in X$. Since $X$ is totally ordered, we have either $x \leq y \leq \varphi(y)=b \leq \varphi(x)$, hence $\varphi(y)=\varphi(x)$ by $(\mathrm{c})$, or $y \leq x \leq b=\varphi(y)$, hence $\varphi(x)=\varphi(y)$ by (c) again. Therefore $b=\varphi(x)$, showing that $[x, \varphi(x)] \cap A=\{\varphi(x)\}$. This proves that (5.5) implies (5.4).

We now fix a map $\varphi: X \rightarrow T$ satisfying (a), (b), (c), and we set

$$
C=\bigcup_{x \in X}[x, \varphi(x)] \quad \text { and } \quad D=\varphi(X)
$$

so that (5.5) becomes $C \cap A=D$. Since $\varphi$ is order-preserving and $\varphi(\hat{0})=\hat{0}$, the set $D$ is a chain in $T$ starting with $\hat{0}$. Let $\bar{C}=C \cup\{\hat{1}\}$ and $\bar{D}=D \cup\{\hat{1}\}$, so that $\bar{D} \in \mathcal{Z}$. Clearly, $\hat{1} \notin C$ if and only if $\hat{1} \notin D$, and therefore the condition $C \cap A=D$ is equivalent to $\bar{C} \cap A=\bar{D}$. It follows that the coefficient of $\varphi$ in (5.3) is equal to

$$
-\sum_{\substack{A \in \mathcal{Z} \\ \sigma_{A} i_{X}=\varphi}} \mu(A, \infty)=-\sum_{\substack{A \in \mathcal{Z} \\ C \cap A=D}} \mu(A, \infty)=-\sum_{\substack{A \in \mathcal{Z} \\ C \cap A=\bar{D}}} \mu(A, \infty),
$$

because the condition $\sigma_{A} i_{X}=\varphi$ is equivalent to (5.5) by the discussion above.

By the defining property of the Möbius function, we obtain

$$
-\sum_{\substack{A \in \mathcal{Z} \\ \bar{C} \cap A=\bar{D}}} \mu(A, \infty)=\sum_{\substack{A, B \in \mathcal{Z} \\ A \subseteq B \\ \bar{C} \cap A=\bar{D}}} \mu(A, B)=\sum_{\substack{B \in \mathcal{Z} \\ D \subseteq B}} \sum_{\substack{A \in \mathcal{Z} \\ A \subseteq B \\ \bar{C} \cap \bar{A}=\bar{D}}} \mu(A, B) .
$$

For a fixed $B \in \mathcal{Z}$, the chain $A$ runs over the interval $[\bar{D}, B]$ with the additional condition $\bar{C} \cap A=\bar{D}$, which can also be written $(\bar{C} \cap B) \cap A=\bar{D}$ because $A \subseteq B$. By a well-known property of the Möbius function (Corollary 3.9.3 in $[\mathrm{St}]$ ), the corresponding sum

$$
\sum_{\substack{A \in[\bar{D}, B] \\(\bar{C} \cap B) \cap A=\bar{D}}} \mu(A, B)
$$

is zero, provided the fixed element $\bar{C} \cap B$ is not equal to the top element $B$. If otherwise $\bar{C} \cap B=B$, then $B \subseteq \bar{C}$ and $A=\bar{C} \cap A=\bar{D}$, so that the sum over $A$ has the single term $\mu(\bar{D}, B)$ for $A=\bar{D}$.

Going back to the coefficient of $\varphi$ in (5.3), we obtain

$$
-\sum_{\substack{A \in \mathcal{Z} \\ \sigma_{A} i_{X}=\varphi}} \mu(A, \infty)=\sum_{\substack{B \in \mathcal{Z} \\ D \subseteq B \subseteq \bar{C}}} \mu(\bar{D}, B)=\sum_{\substack{B \in \mathcal{Z}_{\bar{C}} \\ \bar{D} \subseteq B}} \mu(\bar{D}, B)=-\mu(\bar{D}, \infty)
$$

where the latter symbol $\infty$ denotes a top element added to the poset $\mathcal{Z}_{\bar{C}}$ (consisting of all chains in $\bar{C}$ having least element $\hat{0}$ and greatest element $\hat{1}$ ).

Recall that $\varphi \geq i_{X}$ by condition (b). We now assume that $\varphi>i_{X}$ and we want to prove that $\mu(\bar{D}, \infty)=0$. Let $y \in X$ be minimal such that $\varphi(y)>y$. We claim that, for any $B \in \mathcal{Z}_{\bar{C}}$, the union $B \cup\{y\}$ is totally ordered. We have to prove that any $b \in B$ is comparable with $y$. Since $b \in \bar{C}$, either $b=\hat{1}$ and then we are done because $y \leq \hat{1}$, or there exists $x \in X$ such that $b \in[x, \varphi(x)]$. If $y \leq x$, then $y \leq b$ and we are done again. We can assume now that $y \leq x x$, hence $x<y$ because $X$ is totally ordered. By minimality of $y$, we must have $\varphi(x)=x$, hence $[x, \varphi(x)]=\{x\}$ and $b=x$. It follows that $b<y$. This completes the proof that $B \cup\{y\}$ is totally ordered.

We claim now that $y$ does not belong to $D=\varphi(X)$. Otherwise $y=\varphi(z)$ for some $z \in X$. If we had $z=y$, we would obtain $\varphi(y)=y$, contrary to the choice of $y$. 
It follows that the relation $z \leq \varphi(z)=y$ must be a strict inequality $z<\varphi(z)$. This contradicts the minimality of $y$ and proves the claim. Moreover, $y \notin \bar{D}$ because $y<\varphi(y)$, hence $y \neq \hat{1}$. Consequently, the poset $] \bar{D}, \infty[$ is conically contractible (see 1.5 in $[\mathrm{Qu}]$ ) via the contraction

$$
B \leq B \cup\{y\} \geq \bar{D} \cup\{y\}
$$

and it follows that $\mu(\bar{D}, \infty)=0$.

This shows that the coefficient of $\varphi$ in (5.3) is zero whenever $\varphi>i_{X}$. Therefore we are left with a single term for $\varphi=i_{X}$, namely

$$
f_{\text {tot }} i_{X}=-\mu(\bar{D}, \infty) i_{X} .
$$

But for $\varphi=i_{X}$, we have

$$
C=\bigcup_{x \in X}[x, \varphi(x)]=\bigcup_{x \in X}\{x\}=X=i_{X}(X)=D,
$$

and consequently the only chain in $\bar{C}$ containing $\bar{D}$ is $\bar{D}$ itself. In other words ] $\bar{D}, \infty\left[=\emptyset\right.$ and $\mu(\bar{D}, \infty)=-1$. The required equality $f_{\text {tot }} i_{X}=i_{X}$ follows and this completes the proof of Lemma 5.2.

Proof of Theorem 5.1 : We first show that $f_{\text {tot }}$ is idempotent. Lemma 5.2 implies that $f_{\text {tot }} \sigma_{A}=\sigma_{A}$ for any $A \in \mathcal{Z}$, hence

$$
\left(f_{\text {tot }}\right)^{2}=f_{\text {tot }}\left(-\sum_{A \in \mathcal{Z}} \mu(A, \infty) \sigma_{A}\right)=-\sum_{A \in \mathcal{Z}} \mu(A, \infty) \sigma_{A}=f_{\text {tot }},
$$

as required.

Next we want to pass to opposite lattices and use Lemma 5.2 for $T^{o p}$. To this end, we include $T$ in the notation, so we write $\leq_{T}$ for $\leq$ and we define $f_{\text {tot }}(T)=f_{\text {tot }}$, $\mathcal{E}_{\text {tot }}(T)=\mathcal{E}_{\text {tot }}$, and $\mathcal{Z}(T)=\mathcal{Z}$. Since $\sigma_{A}$ is an endomorphism of $T, \sigma_{A}^{o p}$ is an endomorphism of $T^{o p}$ and we claim that $\sigma_{A}^{o p}=\sigma_{A^{o p}}$, where $A^{o p}$ is equal to $A$, with its opposite order inside $T^{o p}$. In order to prove the claim, we let $s, t \in T$. Clearly $\sigma_{A}(t) \leq_{T} s$ if and only if $s \leq_{T^{o p}} \sigma_{A}(t)$. Since $\sigma_{A}(t) \in A$ and since $\sigma_{A^{o p}}(s)$ is the smallest element of $A^{o p}$ containing $s$ in $T^{o p}$, by (3.6), we get

$s \leq_{T^{o p}} \sigma_{A}(t) \Longleftrightarrow \sigma_{A^{o p}}(s) \leq_{T^{o p}} \sigma_{A}(t) \Longleftrightarrow \sigma_{A}(t) \leq_{T} \sigma_{A^{o p}}(s) \Longleftrightarrow t \leq_{T} \sigma_{A^{o p}}(s)$,

where the latter equivalence comes from the fact that $\sigma_{A^{\circ p}}(s) \in A$ and $\sigma_{A}(t)$ is the smallest element of $A$ containing $t$ in $T$, by (3.6) again. Now the definition of $\sigma_{A}^{o p}$ yields

$$
\sigma_{A}^{o p}(s)=\bigvee_{\substack{t \in T \\ \sigma_{A}(t) \leq_{T} s}} t=\bigvee_{\substack{t \in T \\ t \leq_{T} \sigma_{A^{o p}}(s)}} t
$$

by the above, and the latter element is obviously equal to $\sigma_{A^{\text {op }}}(s)$. Therefore $\sigma_{A}^{o p}=\sigma_{A^{o p}}$, proving the claim.

Now the opposite of our idempotent is

$$
f_{\mathrm{tot}}(T)^{o p}=-\sum_{A \in \mathcal{Z}(T)} \mu(A, \infty) \sigma_{A}^{o p}=-\sum_{A \in \mathcal{Z}(T)} \mu(A, \infty) \sigma_{A^{o p}} .
$$

Using the fact that the passage to opposite chains preserves the inclusion relation between chains, we obtain

$$
f_{\text {tot }}(T)^{o p}=-\sum_{A^{o p} \in \mathcal{Z}\left(T^{o p}\right)} \mu\left(A^{o p}, \infty\right) \sigma_{A^{o p}},
$$

that is, $f_{\text {tot }}(T)^{o p}=f_{\text {tot }}\left(T^{o p}\right)$. 
Lemma 5.2 for the lattice $T^{o p}$ asserts that $f_{\text {tot }}\left(T^{o p}\right) \rho=\rho$ for every $\rho \in \mathcal{E}_{\text {tot }}\left(T^{o p}\right)$. Since $\rho=\psi^{o p}$ for some $\psi \in \mathcal{E}_{\text {tot }}(T)$ (namely $\psi=\rho^{o p}$ ), we get $f_{\text {tot }}(T)^{o p} \psi^{o p}=\psi^{o p}$, that is, $\psi f_{\text {tot }}(T)=\psi$, for all $\psi \in \mathcal{E}_{\text {tot }}$. Together with Lemma 5.2 for $T$, we obtain

$$
\psi f_{\text {tot }}=\psi=f_{\text {tot }} \psi, \quad \forall \psi \in \mathcal{E}_{\text {tot }},
$$

showing that $f_{\text {tot }}$ is the identity element of the two-sided ideal $k \mathcal{E}_{\text {tot }}$.

To prove that $f_{\text {tot }}$ is central in $k \mathcal{E}$, let $\varphi \in \mathcal{E}$. Applying (5.6) to both $f_{\text {tot }} \varphi$ and $\varphi f_{\text {tot }}$ (which belong to $k \mathcal{E}_{\text {tot }}$ ), we see that

$$
f_{\text {tot }} \varphi=f_{\text {tot }} \varphi f_{\text {tot }}=\varphi f_{\text {tot }}
$$

as required. This completes the proof of Theorem 5.1.

5.7. Corollary. There is a two-sided ideal $\mathcal{D}$ of $k \mathcal{E}$ and a ring isomorphism

$$
k \mathcal{E} \cong k \mathcal{E}_{\text {tot }} \times \mathcal{D}
$$

(where $k \mathcal{E}_{\text {tot }}$ is identified with $k \mathcal{E}_{\text {tot }} \times\{0\}$ and $\mathcal{D}$ with $\{0\} \times \mathcal{D}$, as usual).

Proof : By Theorem 5.1, the central idempotent, $f_{\text {tot }}$ generates $k \mathcal{E}_{\text {tot }}$ and therefore the central idempotent id $-f_{\text {tot }}$ generates a complementary two-sided ideal $\mathcal{D}$.

Corollary 5.7 was first proved as Theorem 10.8 in [BT2], by means of a completely different approach which produces a central idempotent $e_{\text {tot }}$ generating the twosided ideal $k \mathcal{E}_{\text {tot }}$.

5.8. Corollary. $e_{\text {tot }}=f_{\text {tot }}$.

Proof : By Theorem 5.1, $f_{\text {tot }}$ is the identity element of the ideal $k \mathcal{E}_{\text {tot. }}$ By Theorem 10.8 in [BT2], $e_{\text {tot }}$ is also the identity element of $k \mathcal{E}_{\text {tot }}$. Therefore they must be equal.

The formula for $e_{\text {tot }}$ given in Theorem 10.8 of [BT2] comes from rather elaborate constructions, which we revisit in Section 6 below.

\section{The original approach to the idempotent}

In this section, we use the original approach of [BT2] and we consider the idempotent $e_{\text {tot }}$, defined in Theorem 10.8 of [BT2] by an explicit, but complicated, formula. Our goal is to give a second proof of Corollary 5.8, namely $e_{\text {tot }}=f_{\text {tot }}$, using the formula for $f_{\text {tot }}$ obtained in Corollary 3.11. In other words, we want to prove the following :

$$
e_{\mathrm{tot}}=-\sum_{A \in \mathcal{Z}} \mu(A, \infty) \sigma_{A},
$$

where we use again the simplified notation of Section 5. We will start from the expression of $e_{\text {tot }}$ given in [BT2]. By using rather delicate arguments, we will show that this expression can be greatly simplified and finally reduces to the right hand side of (6.1).

This second proof of (6.1) is slightly shorter than the proof of Corollary 5.8 obtained by using the developments of Sections 4 and 5 . However, it has the disadvantage of being quite technical, as it uses the cumbersome constructions of [BT2]. Moreover, this proof does not seem to provide useful ideas for proving Conjecture 7.1 below about pole lattices.

We first need to define the notation. For any $n \in \mathbb{N}$, we write $\underline{n}=\{0,1, \ldots, n\}$, a totally ordered lattice with $\hat{0}_{\underline{n}}=0$ and $\hat{1}_{\underline{n}}=n$. We use the set $\mathcal{P}_{n}$ of all chains 
$B=\left\{b_{0}<b_{1}<\ldots<b_{n}=\hat{1}\right\}$ in $T$ whose greatest element is $\hat{1}$. The set $\mathcal{P}_{n}$ parametrizes the set of surjective join-morphisms $\pi: T \rightarrow \underline{n}$ via the rule

$$
b_{i}=\pi^{o p}(i)=\sup \{t \in T \mid \pi(t)=i\} .
$$

Instead of $\underline{n}$, it will be convenient to use a totally ordered lattice $P$ of cardinality $n+1$, that is, a lattice isomorphic to $\underline{n}$, and to define $r(p)=\sup \{q \in P \mid q<p\}$, for any $p \in P-\{\hat{0}\}$. With this notation, a surjective join-morphism $\pi: T \rightarrow P$ corresponds to a chain $B=\left\{b_{p} \mid p \in P\right\}$ defined by

$$
b_{p}=\pi^{o p}(p)=\sup \{t \in T \mid \pi(t)=p\},
$$

and satisfying $b_{p}<b_{q}$ whenever $p<q$. We write $\pi^{B}: T \rightarrow P$ for the surjective join-morphism corresponding to the chain $B \in \mathcal{P}_{n}$. Then $\pi^{B}(t)=\hat{0}$ if $t \leq b_{0}$ and otherwise we recall the rule

$$
\pi^{B}(t)=p \quad \text { if } t \leq b_{p} \text { and } t \not \leq b_{r(p)} .
$$

For any given $B \in \mathcal{P}_{n}$, we choose an element $a_{p} \in\left[b_{r(p)}, b_{p}\right]$ for each $p \in P-\{\hat{0}\}$. This defines a family $A=\left(a_{p}\right)_{p \in P-\{\hat{0}\}}$ of elements of $T$. We let $\mathcal{J}_{B}$ be the set of all families $A=\left(a_{p}\right)_{p \in P-\{\hat{0}\}}$ of elements of $T$ such that $a_{p} \in\left[b_{r(p)}, b_{p}\right]$ for every $p \in P-\{\hat{0}\}$. If $A \in \mathcal{J}_{B}$, we also set $a_{\hat{0}}=\hat{0}$ and we define

$$
j_{A}^{B}: P \longrightarrow T, \quad j_{A}^{B}(p)=a_{p} .
$$

Clearly $j_{A}^{B}$ is order-preserving (because if $p<q$ in $P$, then $p \leq r(q)$, hence $a_{p} \leq$ $\left.b_{p} \leq b_{r(q)} \leq a_{q}\right)$, and it also maps $\hat{0}$ to $\hat{0}$. Therefore $j_{A}^{B}$ is a join-morphism.

Now let $B^{-}=\left\{b_{r(p)} \mid p \in P-\{\hat{0}\}\right\}$ and for any $A \in \mathcal{J}_{B}$, write

$$
\mu\left(B^{-}, A\right)=\prod_{p \in P-\{\hat{0}\}} \mu\left(b_{r(p)}, a_{p}\right),
$$

where $\mu\left(b_{r(p)}, a_{p}\right)$ denotes the Möbius function for the lattice $T$. Now we allow the family $A$ to vary (i.e. $a_{p}$ varies in $\left[b_{r(p)}, b_{p}\right]$ for each $p \neq \hat{0}$ ) and we define

$$
j^{B}=(-1)^{n} \sum_{A \in \mathcal{J}_{B}} \mu\left(B^{-}, A\right) j_{A}^{B} \in \operatorname{Hom}_{k \mathcal{L}}(P, T) .
$$

By Proposition 10.2 of [BT2], $f_{B}=j^{B} \pi^{B}$ is an idempotent in $\mathcal{E}$ and when $n \geq 0$ varies and $B \in \mathcal{P}_{n}$ varies, the idempotents $f_{B}$ are pairwise orthogonal (Corollary 10.5 of $[\mathrm{BT} 2])$. The idempotent $e_{\mathrm{tot}}$ is then defined by the formula

$$
e_{\text {tot }}=\sum_{n=0}^{N} \sum_{B \in \mathcal{P}_{n}} f_{B},
$$

see Theorem 10.8 of [BT2]. For each $B \in \mathcal{P}_{n}$, the idempotent $f_{B}$ is a linear combination of join-morphisms $j_{A}^{B} \pi^{B}$. We are going to prove that most of these join-morphisms cancel pairwise in the sum

$$
e_{\text {tot }}=\sum_{n=0}^{N} \sum_{B \in \mathcal{P}_{n}}(-1)^{n} \sum_{A \in \mathcal{J}_{B}} \mu\left(B^{-}, A\right) j_{A}^{B} \pi^{B} .
$$

More precisely, we consider all triples $\left\{(n, B, A) \mid n \in \mathbb{N}, B \in \mathcal{P}_{n}, A \in \mathcal{J}_{B}\right\}$ such that $a_{x}<b_{x}$ for some $x \in P$. For such a triple, we let $p \in P$ be minimal with respect to the condition $a_{p}<b_{p}$. Since $a_{p} \in\left[b_{r(p)}, b_{p}\right]$ for $p \neq \hat{0}$, we can either have $b_{r(p)}=a_{p}<b_{p}$ or $b_{r(p)}<a_{p}<b_{p}$. The case $p=\hat{0}$ is special because we always have $a_{\hat{0}}=\hat{0}$. It follows that $p$ must satisfy one of the following 4 cases :

A1. $p \neq \hat{0}, r(p) \neq \hat{0}, b_{r(p)}=a_{p}<b_{p}$, and $a_{x}=b_{x}$ for any $x<p$.

A2. $p \neq \hat{0}, r(p)=\hat{0}, b_{r(p)}=a_{p}<b_{p}$, and $\hat{0}=a_{\hat{0}}=b_{\hat{0}}$. 
B1. $p \neq \hat{0}, b_{r(p)}<a_{p}<b_{p}$, and $a_{x}=b_{x}$ for any $x<p$.

B2. $p=\hat{0}$ and $\hat{0}=a_{\hat{0}}<b_{\hat{0}}$.

We now examine each of these cases successively.

Case A1. Suppose we are in Case A1. Define

$$
\widetilde{P}=P-\{p\}, \quad \widetilde{b}_{q}=b_{q} \forall q \in \widetilde{P}-\{r(p)\}, \quad \widetilde{b}_{r(p)}=b_{p} .
$$

This defines a chain $\widetilde{B}$ in $T$ and a surjective join-morphism $\pi^{\widetilde{B}}: T \rightarrow \widetilde{P}$, satisfying in particular $\pi^{\widetilde{B}}\left(b_{p}\right)=r(p)$. Let $\widetilde{A} \in \mathcal{J}_{\widetilde{B}}$ be the family defined by

$$
\widetilde{a}_{q}=a_{q} \forall q \in \widetilde{P}-\{r(p)\}, \quad \widetilde{a}_{r(p)}=b_{r(p)},
$$

and let $j_{\widetilde{A}}^{\widetilde{B}}: \widetilde{P} \rightarrow T$ be the corresponding join-morphism. Then we obtain

$$
b_{r(r(p))}<b_{r(p)}<b_{p}, \quad \text { that is, } \quad \widetilde{b}_{r(r(p))}<\widetilde{a}_{r(p)}<\widetilde{b}_{r(p)},
$$

so that $\widetilde{P}$ and its element $r(p)$ are in Case B1 (because $r(p) \neq \hat{0}$ by assumption A1). Moreover, $j_{A}^{B} \pi^{B}=j_{\widetilde{A}}^{\widetilde{B}} \pi^{\widetilde{B}}$. This is easy to check on most elements of $T$, the only nontrivial case being

$$
j_{A}^{B} \pi^{B}\left(b_{p}\right)=j_{A}^{B}(p)=a_{p}=b_{r(p)}=\widetilde{a}_{r(p)}=j_{\widetilde{A}}^{\widetilde{B}}(r(p))=j_{\widetilde{A}}^{\widetilde{B}} \pi^{\widetilde{B}}\left(b_{p}\right) .
$$

Finally, since $\mu\left(b_{r(p)}, a_{p}\right)=\mu\left(b_{r(p)}, b_{r(p)}\right)=1$, the coefficient of $j_{A}^{B} \pi^{B}$ is equal to

$$
\begin{aligned}
(-1)^{n} \mu\left(B^{-}, A\right) & =(-1)^{n} \prod_{x \in P-\{\hat{0}\}} \mu\left(b_{r(x)}, a_{x}\right) \\
& =(-1)^{n} \prod_{x \in P-\{\hat{0}, p\}} \mu\left(b_{r(x)}, a_{x}\right) \\
& =(-1)^{n} \prod_{x \in \widetilde{P}-\{\hat{0}\}} \mu\left(\widetilde{b}_{r(x)}, \widetilde{a}_{x}\right) \\
& =-(-1)^{n-1} \mu\left(\widetilde{B}^{-}, \widetilde{A}\right),
\end{aligned}
$$

using the fact that, for $x=r(p)$, we have $\widetilde{a}_{r(p)}=b_{r(p)}$ and also $a_{r(p)}=b_{r(p)}$ by minimality of the choice of $p$. This shows that

$$
(-1)^{n} \mu\left(B^{-}, A\right) j_{A}^{B} \pi^{B} \quad \text { and } \quad(-1)^{n-1} \mu\left(\widetilde{B}^{-}, \widetilde{A}\right) j_{\widetilde{A}}^{\widetilde{B}} \pi^{\widetilde{B}}
$$

cancel in the sum (6.2). Thus any Case A1 cancels with some Case B1.

Case B1. Suppose we are in Case B1. Define

$$
\widehat{P}=P_{<p} \sqcup\{s\} \sqcup P_{\geq p},
$$

with the total order defined by $x<s$ for all $x \in P_{<p}$ and $s<x$ for all $x \in P_{\geq p}$, so that $r(p)=s$. Moreover, define

$$
\widehat{b}_{q}=b_{q} \forall q \in \widehat{P}-\{s\}, \quad \widehat{b}_{s}=a_{p} .
$$

This defines a chain $\widehat{B}$ in $T$ and a surjective join-morphism $\pi^{\widehat{B}}: T \rightarrow \widehat{P}$, satisfying in particular $\pi^{\widehat{B}}\left(a_{p}\right)=s$ and $\pi^{\widehat{B}}\left(b_{p}\right)=p$. Finally, let $\widehat{A} \in \mathcal{J}_{\widehat{B}}$ be the family defined by

$$
\widehat{a}_{q}=a_{q} \forall q \in \widehat{P}-\{s\}, \quad \widehat{a}_{s}=a_{p},
$$

and let $j_{\widehat{A}}^{\widehat{B}}: \widehat{P} \rightarrow T$ be the corresponding join-morphism. Then we obtain

$$
a_{p}<b_{p}, \quad \text { that is, } \quad \widehat{b}_{r(p)}=\widehat{a}_{p}<\widehat{b}_{p},
$$

so that $\widehat{P}$ and its element $p$ are in Case A1 (because $r(p)=s \neq \hat{0}$ ). Applying the procedure described in Case A1, we note that $\widehat{P}-\{p\}$ is isomorphic to $P$ and it 
follows easily that we recover the Case B1 we started with. Thus every Case B1 has been canceled with a corresponding Case A1.

Case A2. Suppose we are in Case A2. Since $r(p)=\hat{0}, p$ is the least element of $P-\{\hat{0}\}$. Define

$$
\widetilde{P}=P-\{p\}, \quad \widetilde{b}_{q}=b_{q} \forall q \in \widetilde{P}-\{\hat{0}\}, \quad \widetilde{b}_{\hat{0}}=b_{p} .
$$

This defines a chain $\widetilde{B}$ in $T$ and a surjective join-morphism $\pi^{\widetilde{B}}: T \rightarrow \widetilde{P}$, satisfying in particular $\pi^{\widetilde{B}}\left(b_{p}\right)=\hat{0}$. Let $\widetilde{A} \in \mathcal{J}_{\widetilde{B}}$ be the family defined by

$$
\widetilde{a}_{q}=a_{q} \forall q \in \widetilde{P}-\{\hat{0}\}, \quad \widetilde{a}_{\hat{0}}=b_{\hat{0}},
$$

and let $j_{\widetilde{A}}^{\widetilde{B}}: \widetilde{P} \rightarrow T$ be the corresponding join-morphism. We have $\hat{0}=b_{\hat{0}}$ by minimality of $p$ and we obtain

$$
\hat{0}=b_{\hat{0}}<b_{p}, \quad \text { that is, } \quad \hat{0}=\widetilde{a}_{\hat{0}}<\widetilde{b}_{\hat{0}},
$$

so that $\widetilde{P}$ and its element $\hat{0}$ are in Case B2. The argument for the Möbius function holds in the same way as in Case A1 and it follows that any Case A2 cancels with some Case B2 in the sum (6.2).

Case B2. Suppose we are in Case B2. Define

$$
\widehat{P}=\{s\} \sqcup P,
$$

with the total order defined by $s<x$ for all $x \in P$, so that $r(p)=s=\hat{0}_{\widehat{P}}$. Moreover, define

$$
\widehat{b}_{q}=b_{q} \forall q \in P, \quad \widehat{b}_{s}=\hat{0} .
$$

This defines a chain $\widehat{B}$ in $T$ and a surjective join-morphism $\pi^{\widehat{B}}: T \rightarrow \widehat{P}$, satisfying in particular $\pi^{\widehat{B}}\left(b_{p}\right)=p$. Finally, let $\widehat{A} \in \mathcal{J}_{\widehat{B}}$ be the family defined by

$$
\widehat{a}_{q}=a_{q} \forall q \in P, \quad \widehat{a}_{p}=\hat{0},
$$

and let $j_{\widehat{A}}^{\widehat{B}}: \widehat{P} \rightarrow T$ be the corresponding join-morphism. Then we obtain

$$
\hat{0}<b_{p}, \quad \text { that is, } \quad \hat{0}=\widehat{a}_{\hat{0}}=\widehat{b}_{\hat{0}}=\widehat{a}_{p}<\widehat{b}_{p},
$$

so that $\widehat{P}$ and its element $p$ are in Case A2. Applying the procedure described in Case A2, we note that $\widehat{P}-\{p\}$ is isomorphic to $P$ and it follows easily that we recover the Case B2 we started with. Thus every Case B2 has been canceled with a corresponding Case A2.

Applying the cancelations described above, we can now eliminate all the joinmorphisms $j_{A}^{B} \pi^{B}$ corresponding to a triple $\left(n \in \mathbb{N}, B \in \mathcal{P}_{n}, A \in \mathcal{J}_{B}\right)$ satisfying $a_{x}<b_{x}$ for some $x \in P$. We are left with the triples satisfying $a_{x}=b_{x}$ for all $x \in P$. In such a case, we have $b_{\hat{0}}=\hat{0}$, that is, $\hat{0} \in B$, hence $B \in \mathcal{Z}_{n}$, where $\mathcal{Z}_{n}$ is the set of chains described at the beginning of Section 3. Thus the second sum in (6.2) runs over $B \in \mathcal{Z}_{n}$. Moreover, $A=B-\{\hat{0}\}=: B^{+}$and so the third sum in $(6.2)$ reduces to a single term.

Now we have $j_{B^{+}}^{B} \pi^{B}(t)=b_{p}$ if $\pi^{B}(t)=p$, that is, if $t \leq b_{p}$ but $t \not \leq b_{r(p)}$. In other words,

$$
j_{B^{+}}^{B} \pi^{B}(t)=\min \{b \in B \mid t \leq b\}
$$

and this is exactly the definition of the endomorphism $\sigma_{B}$ considered in Section 3 , see the formula (3.6). Thus

$$
j_{B^{+}}^{B} \pi^{B}=\sigma_{B} \quad \forall B \in \mathcal{Z}_{n} .
$$


Moreover, the coefficient of $j_{B^{+}}^{B} \pi^{B}$ in the expression for $e_{\text {tot }}$ is the Möbius function

$$
(-1)^{n} \mu\left(B^{-}, B^{+}\right)=(-1)^{n} \prod_{p \in P-\{\hat{0}\}} \mu\left(b_{r(p)}, b_{p}\right)=-\mu_{\mathcal{Z}}(B, \infty),
$$

by Lemma 3.12, where the latter Möbius function is the Möbius function of the poset $\mathcal{Z} \sqcup\{\infty\}$. It follows that the expression for $e_{\text {tot }}$ given in (6.2) reduces to

$$
e_{\text {tot }}=\sum_{n=0}^{N} \sum_{B \in \mathcal{Z}_{n}}(-1) \mu_{\mathcal{Z}}(B, \infty) \sigma_{B}=-\sum_{B \in \mathcal{Z}} \mu_{\mathcal{Z}}(B, \infty) \sigma_{B} .
$$

This completes the proof of (6.1).

6.3. Remark. It is proved in Theorem 10.6 of [BT2] that the two-sided ideal $k \mathcal{E}_{\text {tot }}$ is isomorphic to a product of matrix algebras

$$
k \mathcal{E}_{\mathrm{tot}} \cong \prod_{n=0}^{N} M_{\left|\mathcal{Z}_{n}\right|}(k)
$$

where $N$ is the maximal length of a chain in $T$. It should be noticed that the new approach to the generating idempotent of $k \mathcal{E}_{\text {tot }}$ explained in the present paper does not simplify in any way the proof of this result. In particular, if $T$ is totally ordered, then

$$
k \mathcal{E}=k \mathcal{E}_{\text {tot }} \cong \prod_{n=0}^{N} M_{\left|\mathcal{Z}_{n}\right|}(k)
$$

and this is a semi-simple algebra whenever $k$ is a field. As noticed in Remark 11.3 of [BT2], this result is similar, but not equivalent, to a theorem proved in $[\mathrm{FHH}]$ about the planar rook algebra.

\section{The case of pole lattices}

Pole lattices are generalizations of totally ordered lattices and are defined in Section 2. They play a role in our work [BT5] on correspondence functors because they have a property of semi-simplicity analogous to the results of Remark 6.3.

We consider now the endomorphism $f_{\text {pole }}$ obtained by using the family of pole lattices instead of totally ordered lattices. Recall from Definition 2.2 that

$$
f_{\text {pole }}:=-\sum_{\alpha \in \mathcal{H}_{\text {pole }}} \mu_{\mathcal{H}_{\text {pole }}}(-\infty, \alpha) \alpha,
$$

where $\mathcal{H}_{\text {pole }}=\{\alpha \in \mathcal{E} \mid \alpha \geq$ id, $\alpha(T) \in$ pole $\}$. Recall also that $k \mathcal{E}_{\text {pole }}$ denotes the two-sided ideal generated by all endomorphisms $\alpha \in \mathcal{E}$ whose image $\alpha(T)$ is a pole lattice. In analogy with Theorem 5.1, we conjecture the following :

7.1. Conjecture. Let $f_{\text {pole }}$ be as as above.

(a) $f_{\text {pole }}$ is a central idempotent of $k \mathcal{E}$.

(b) $f_{\text {pole }}$ is the identity element of the ideal $k \mathcal{E}_{\text {pole }}$.

By Theorem 8.3 of [BT5], we know that $k \mathcal{E}=k \mathcal{E}_{\text {pole }} \oplus \mathcal{D}^{\prime}$ for some two-sided ideal $\mathcal{D}^{\prime}$. The projection of id onto the first factor is an idempotent $e_{\text {pole }}$ satisfying the two properties of the Conjecture, but constructed in a very different way. Thus Conjecture 7.1 asserts in fact that $f_{\text {pole }}=e_{\text {pole }}$.

By using the computer system [GAP4], we can prove that Conjecture 7.1 holds in very many cases. Actually, the idea of the formula in Definition 2.2 slowly emerged in 2018 from complicated calculations using already the GAP software 
[GAP4], based on existing lists of finite posets (either from a personal database or from $[\mathrm{Mc}])$.

In order to verify the conjecture, we consider a finite partially ordered set $E$ and the lattices admitting $E$ as subposet of join-irreducible elements. As there are in general too many such lattices for any given $E$, we deal only with two of them. The largest one is the distributive lattice $D(E)$ of all lower subsets of $E$. There is also a smallest such lattice $K(E)$, which is not hard to construct as the image of a suitable closure operator in the lattice $D(E)$.

We have checked Conjecture 7.1 for $D(E)$ and $|E| \leq 5$, and for $K(E)$ and $|E| \leq 8$. In the case $|E|=8$, two weeks were needed for running the program for $K(E)$. In the next case, there are so many posets $E$ of cardinal 9 that running the program for $K(E)$ and $|E|=9$ would require too much time.

7.2. Remark. In the case of totally ordered lattices, we were able to replace $\mathcal{H}_{\text {tot }}$ by the subposet $\mathcal{G}_{\text {tot }}$ of all $\alpha \in \mathcal{H}_{\text {tot }}$ satisfying the additional condition $\alpha^{2}=\alpha$. This was achieved by means of a homotopy equivalence $\mathcal{G}_{\text {tot },<\tau} \hookrightarrow \mathcal{H}_{\text {tot, }<\tau}$ (see Lemma 4.2). No such reduction is possible in the case of pole lattices, because in the sum defining $f_{\text {pole }}$ it may happen that some terms $\alpha$ are not idempotent (i.e. $\left.\alpha^{2} \neq \alpha\right)$. The first instance of this occurs with the lattice

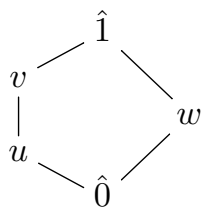

If $[x, y, z]$ denotes the endomorphism of this lattice such that $u \mapsto x, v \mapsto y, w \mapsto z$, we obtain

$$
f_{\text {pole }}=[v, \hat{1}, \hat{1}]-[u, \hat{1}, \hat{1}]-[v, v, \hat{1}]-[v, \hat{1}, w]+[u, v, \hat{1}]+[u, \hat{1}, w]+[v, v, w]
$$

and neither $[v, \hat{1}, \hat{1}]$ nor $[v, \hat{1}, w]$ are idempotent.

On the other hand, we easily get

$$
f_{\text {tot }}=-[\hat{1}, \hat{1}, \hat{1}]+[u, v, \hat{1}]+[\hat{1}, \hat{1}, w] .
$$

7.3. Remark. One may wonder if the definition of $f_{\mathcal{F}}$ for other families $\mathcal{F}$ gives rise to a central idempotent generating the ideal $k \mathcal{E}_{\mathcal{F}}$. Despite various attempts, we could not find any sufficient evidence for a result of this kind. There is a positive answer in many small cases, but also a negative one in some easy cases, such as the family $\operatorname{tot}(r)$ of all totally ordered lattices of size $\leq r$. The two-sided ideal $k \mathcal{E}_{\text {tot }(r)}$ is generated by a central idempotent, but its expression may involve endomorphisms $\alpha$ of $T$ which do not satisfy the condition $\alpha \geq$ id. This is obvious when $r=1$ since the constant map $c_{\hat{0}}$ onto $\hat{0}$ is the only join-endomorphism of $T$ whose image is totally ordered of size 1 (so that $k \mathcal{E}_{\text {tot(1) }}$ has rank one), but $c_{\hat{0}} \nsucceq$ id (whenever $|T| \geq 2)$.

\section{References}

[BT1] S. Bouc, J. Thévenaz. Correspondence functors and finiteness conditions, J. Algebra 495 (2018), 150-198.

[BT2] S. Bouc, J. Thévenaz. Correspondence functors and lattices, J. Algebra 518 (2019), 453-518. [BT3] S. Bouc, J. Thévenaz. The algebra of Boolean matrices, correspondence functors, and simplicity, J. Comb. Algebra 4 (2020), 215-267. 
[BT4] S. Bouc, J. Thévenaz. Tensor product of correspondence functors, J. Algebra 558 (2020), 146-175.

[BT5] S. Bouc, J. Thévenaz. Simple and projective correspondence functors, Representation Theory 25 (2021), 224-264.

[FHH] D. Flath, T. Halverson, K. Herbig. The planar rook algebra and Pascal's triangle, Enseign. Math. 55 (2009), no. 1-2, 77-92.

[GAP4] The GAP Group. GAP - Groups, Algorithms, and Programming, Version 4.11.0, 2020. http://www .gap-system.org

[Mc] B. D. McKay. Digraphs.

http://users. cecs.anu.edu.au/bdm/data/digraphs.html

$[\mathrm{Qu}] \mathrm{D}$. Quillen, Homotopy properties of the poset of nontrivial $p$-subgroups of a group, Adv. Math. 28 (1978), 101-128.

[St] R. P. Stanley. Enumerative Combinatorics, Vol. I, Second edition, Cambridge studies in advanced mathematics 49, Cambridge University Press, 2012. 